\title{
Reverse right ventricular remodeling after pulmonary endarterectomy in patients with chronic thromboembolic pulmonary hypertension: Utility of magnetic resonance imaging to demonstrate restoration of the right ventricle
}

Herre J. Reesink, MD, ${ }^{\text {a } J . ~ T i m ~ M a r c u s, ~ P h D, ~}{ }^{\text {b }}$ Igor I. Tulevski, MD, PhD, ${ }^{c}$ Stuart Jamieson, MD, ${ }^{d}$ Jaap J. Kloek, MD, Anton Vonk Noordegraaf, MD, PhD, ${ }^{f}$ and Paul Bresser, MD, PhD ${ }^{a}$

From the Departments of Pulmonology, Cardiology, ${ }^{\mathrm{c}}$ and Cardiothoracic Surgery ${ }^{\mathrm{e}}$ of the Academic Medical Center, University of Amsterdam, and the Departments of Physics and Medical Technology ${ }^{\mathrm{b}}$ and Pulmonology ${ }^{\mathrm{f}}$ of the Free University Medical Center, Amsterdam, The Netherlands, and from the Department of Cardiothoracic Surgery of the University of California San Diego, San Diego, Calif. ${ }^{\mathrm{d}}$

Herre J. Reesink was supported by a grant from Actelion Pharmaceuticals Nederland bv, Woerden, The Netherlands.

Received for publication March 31, 2006; revisions received Aug 14, 2006; accepted for publication Sept 11, 2006.

Address for reprints: P. Bresser, MD, PhD, Academic Medical Center, University of Amsterdam, Department of Pulmonology, F5-144, PO Box 22700, 1100 DE Amsterdam, the Netherlands (E-mail: P.Bresser@ amc.uva.nl).

J Thorac Cardiovasc Surg 2007;133:58-64 $0022-5223 / \$ 32.00$

Copyright $\odot 2007$ by The American Association for Thoracic Surgery

doi:10.1016/j.jtcvs.2006.09.032
Objectives: Pulmonary arterial hypertension causes right ventricular remodeling; that is, right ventricular dilatation, hypertrophy, and leftward ventricular septal bowing. We studied the effect of pulmonary endarterectomy on the restoration of right ventricular remodeling in patients with chronic thromboembolic pulmonary hypertension by magnetic resonance imaging.

Methods: In 17 patients with chronic thromboembolic pulmonary hypertension, before and at least 4 months after pulmonary endarterectomy, and in 12 healthy controls, right ventricular and left ventricular end-diastolic and end-systolic volumes (milliliters) and mass (grams per meter squared) and leftward ventricular septal bowing ( 1 divided by the radius of curvature in centimeters) were determined by magnetic resonance imaging.

Results: Before pulmonary endarterectomy, right ventricular volumes, left ventricular end-diastolic volume, right ventricular mass, and leftward ventricular septal bowing differed significantly between patients with chronic thromboembolic pulmonary hypertension and healthy control subjects. After pulmonary endarterectomy, pulmonary hemodynamics improved, and right and left ventricular volumes and leftward ventricular septal bowing normalized; right ventricular mass decreased significantly $\left(46 \pm 14\right.$ to $31 \pm 9 \mathrm{~g} \cdot \mathrm{m}^{-2}, P<.0005$ ), but did not completely normalize. The change in total pulmonary resistance correlated with the change in right ventricular ejection fraction $(r=0.50, P<.05)$, right ventricular mass $(r=$ $0.63, P<.01)$, and leftward ventricular septal bowing $(r=0.50, P<.05)$.

Conclusions: Right ventricular remodeling was observed in patients with chronic thromboembolic pulmonary hypertension and restored almost completely after a hemodynamically successful pulmonary endarterectomy. Magnetic resonance imaging is a valuable tool to evaluate cardiac remodeling and function in patients with chronic thromboembolic pulmonary hypertension, both before and after pulmonary endarterectomy.

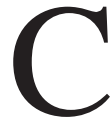
hronic thromboembolic pulmonary hypertension (CTEPH) results from incomplete resolution of the vascular obstruction caused by pulmonary thromboemboli. ${ }^{1}$ If left untreated, over time, a gradual hemodynamic and symptomatic decline can be observed in these patients. ${ }^{2}$ As a consequence, prognosis in CTEPH is poor and proportional to the degree of pulmonary hypertension. ${ }^{3}$ Advanced CTEPH leads to cardiac remodeling, involving right ventricular (RV) dilatation and hypertrophy, tricuspid regurgitation, and leftward ventricular septal bowing (LVSB), with consequent impact on cardiac function. As a consequence, death in most patients with CTEPH is caused by progressive RV failure. Pulmonary 


$$
\begin{aligned}
& \text { Abbreviations and Acronyms } \\
& \begin{aligned}
\text { CTEPH }=\text { chronic thromboembolic pulmonary } & \text { hypertension } \\
\text { EDV } & =\text { end-diastolic volume } \\
\mathrm{EF} & =\text { ejection fraction } \\
\mathrm{ESV} & =\text { end-systolic volume } \\
\mathrm{LV} & =\text { left ventricle (ventricular) } \\
\mathrm{LVSB} & =\text { leftward ventricular septal bowing } \\
\mathrm{mPAP} & =\text { mean pulmonary artery pressure } \\
\mathrm{MRI} & =\text { magnetic resonance imaging } \\
\mathrm{PEA} & =\text { pulmonary endarterectomy } \\
\mathrm{RV} & =\text { right ventricle (ventricular) } \\
\mathrm{TPR} & =\text { total pulmonary resistance }
\end{aligned}
\end{aligned}
$$

endarterectomy (PEA) represents the therapy of choice for patients with surgically accessible thrombi. ${ }^{1,4}$ PEA may be performed with an acceptable mortality risk and results in clinical improvement and often normalization of pulmonary hemodynamics. ${ }^{1,5}$

Magnetic resonance imaging (MRI) is a highly accurate method to quantify RV function and dimensions in patients with CTEPH. ${ }^{6,7}$ In addition, RV hypertrophy ${ }^{8,9}$ and $\mathrm{LVSB}^{10}$ can be adequately quantified by MRI. Restoration of cardiac function within 2 weeks after PEA was described recently by Kreitner and coworkers. ${ }^{6}$ However, restoration of cardiac remodeling after PEA has not been previously studied. The aim of the present study was to determine restoration of $\mathrm{RV}$ remodeling in patients with CTEPH after PEA by means of MRI.

\section{Materials and Methods Study Subjects}

Seventeen ( 8 male; $49 \pm 14$ years, range 29-70 years) of 22 consecutive patients with a diagnosis of CTEPH, referred to the Academic Medical Center of the University of Amsterdam, were studied before and after PEA. Two patients refused to participate because of claustrophobia, 1 patient died postoperatively, and 2 patients refused to undergo a second MRI after surgery. Diagnosis of CTEPH and eligibility for PEA were established on the basis of previously reported procedures and criteria. ${ }^{11}$ Diagnosis and cardiopulmonary hemodynamics were determined by pulmonary angiography and right heart catheterization. Postoperative hemodynamics was determined on the first or second day after PEA, before removal of the Swan-Ganz catheter (Edwards LifeSciences, Irvine, Calif). Coronary angiography was routinely performed in all patients older than 50 years of age, and in patients older than 40 years of age if they had a history of smoking.

Twelve healthy volunteers ( 5 male; $42 \pm 15$ years, range 21-60 years) served as controls for the RV and left ventricular (LV) volumes, mass, and septal bowing. All patients and controls gave informed consent to the study protocol, which was approved by the local ethical committee.

\section{MRI Measurement}

MRI was performed with a 4-element body phased-array coil and a 1.5-T whole body system (Sonata, Siemens Medical Solutions, Erlangen, Germany). MRI measurements were obtained before and at least 4 months after PEA.

MRI breath-hold cine imaging was electrocardiographically triggered and performed in the cardiac short-axis view in a stack of parallel imaging planes covering the LV and RV from base to apex. In 11 patients, for both studies, a spoiled gradient echo sequence was used as specified by Kreitner and coworkers. ${ }^{6}$ In 6 patients, for both studies, a steady-state free precession MRI pulse sequence was applied (also denoted by balanced Fast Field Echo), with 11-phase-ending lines (ky) lines per heartbeat, repetition time $/$ echo time $/$ alpha $=34 \mathrm{~ms} / 1.6 \mathrm{~ms} / 60^{\circ}$. Pixel size was $1.3 \times$ $1.3 \mathrm{~mm}$, slice thickness $6 \mathrm{~mm}$, and slice distance $4 \mathrm{~mm} .{ }^{12}$ In all patients, subsequent analysis was performed with the MR Analytical Software System (Medis, Leiden, The Netherlands). From the short-axis cine images, the RV and LV volumes were calculated for each temporal frame in the cardiac cycle. The end-diastolic (EDV) and end-systolic volumes (ESV) were assessed from the stack of parallel short-axis images, and ejection fraction (EF) and stroke volume were subsequently calculated. RV and LV myocardial masses were assessed from the stack of parallel short-axis images by manual detection of endocardial and epicardial borders on each slice using the MR Analytical Software System (Medis). Cardiac volume and mass were corrected for body surface area. The septal curvature was evaluated for the short-axis image plane at midventricular level (at least one papillary muscle visible). The cine time frame, used for measuring the septal curvature, was in early diastole, that is, the temporal frame after aortic valve closure where the LVSB was most manifest (for this frame, the delay after the R-wave trigger was in the range from 320 to $540 \mathrm{~ms}$ ). In the volunteers, who showed no LVSB, the second time frame after aortic valve closure was chosen. Septal bowing was quantified by the curvature (defined as 1 divided by the radius of curvature in centimeters) as described previously. ${ }^{10}$ Positive values of this curvature ratio denote (physiologic) rightward ventricular septal bowing, and negative values denote LVSB. An additional 3-chamber long-axis cine was acquired to measure the timing of aortic valve closure.

\section{Surgical Procedure}

PEA was performed according to the protocol of the University of California San Diego. ${ }^{2,13}$ PEA is performed via median sternotomy. After initiation of cardiopulmonary bypass, during deep hypothermia $\left(20^{\circ} \mathrm{C}\right)$, the right pulmonary artery is incised where it passes the aorta to the division of the lower lobe arteries. On the left, the incision extends from the main pulmonary artery to the origin of the left upper lobe branch. The organized thromboembolic material is fibrotic and adherent to the vessel wall. An endarterectomy plane is established between the intima and the fibrotic thromboembolic material. Subsequently, the obstructing material is grasped with a forceps, and distal, circumferential dissection is performed with an aspirating dissector. Circulatory arrest is mandatory to ensure optimal visibility in the presence of usually copious retrograde blood flow from a hypertrophied bronchial circulation. The circulatory arrest period is limited to 20 minutes, with restoration of flow between each arrest. 

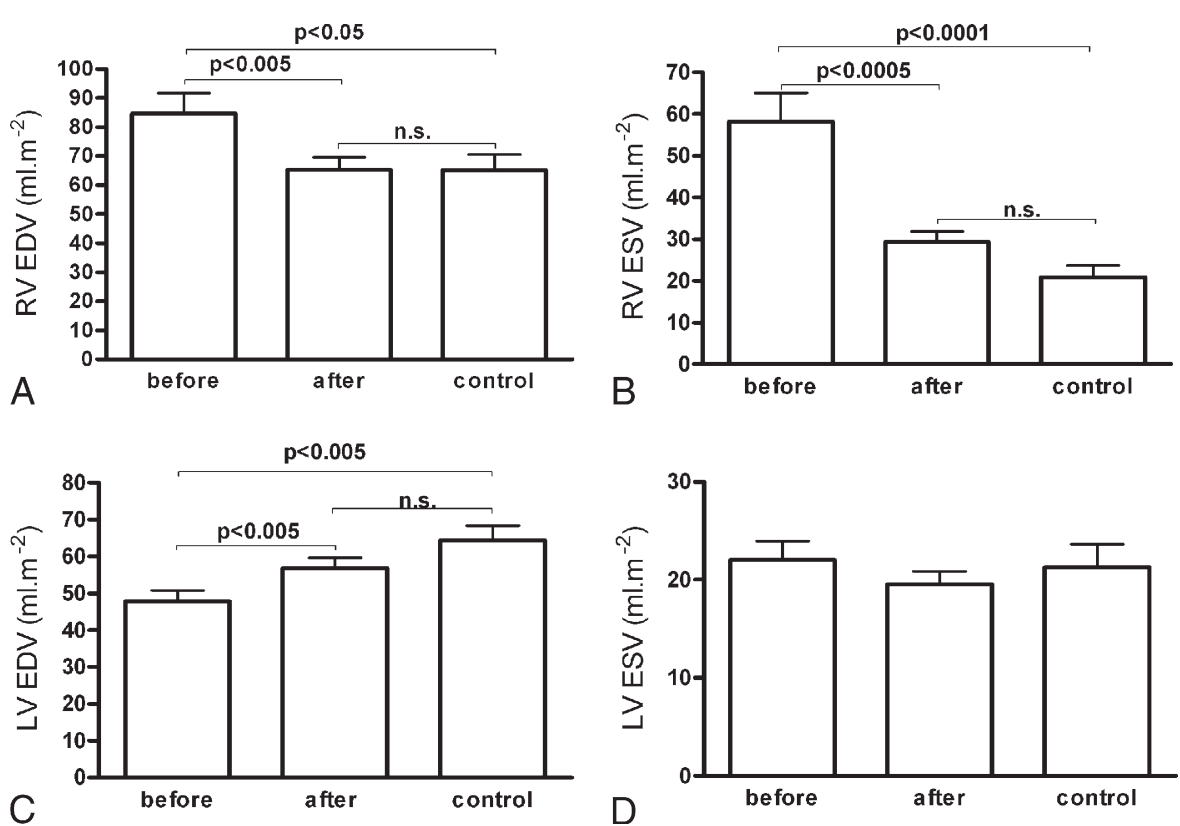

Figure 1. RVEDV (A), RVESV (B), LVEDV (C), and LVESV (D) in patients with CTEPH (n = 17) before and after PEA compared with healthy controls $(n=12)$. Data are expressed as mean $\pm S E M$. n.s., Not significant.

\section{Statistical Analysis}

All data are expressed as mean \pm standard deviation, unless otherwise specified. All analyses were performed with a statistical package (SPSS 11.5; SPSS, Inc, Chicago, Ill). The Spearman rank correlation test was used to assess correlations between ventricular dimensions and the hemodynamic parameters and was tested for 2-sided significance. The Wilcoxon signed rank test was used to analyze the differences between preoperative and postoperative parameters. The Mann-Whitney test was used to analyze differences between patients with and without residual pulmonary hypertension after PEA and between patients and healthy controls.

\section{Results}

Preoperatively, the mean pulmonary artery pressure $(\mathrm{mPAP})$ was $51 \pm 11 \mathrm{~mm} \mathrm{Hg}$, the cardiac index $1.85 \pm 0.45$ $\mathrm{L} \cdot \min \cdot \mathrm{m}^{-2}$, and the total pulmonary resistance (TPR) $1131 \pm 476$ dynes $\cdot \mathrm{s} \cdot \mathrm{cm}^{-5}$. Right atrial and pulmonary capillary wedge pressures were $11 \pm 5$ and $9 \pm 3 \mathrm{~mm} \mathrm{Hg}$, respectively. RV and $\mathrm{LV}$ dysfunction, as defined by an EF less than $45 \%$, was present in 15 and 3 patients, respectively. Coronary artery disease was not present in any of the patients studied.

\section{Effect of PEA}

PEA resulted in substantial hemodynamic improvement in all patients. Postoperatively, 2 days after PEA, mPAP had decreased to $26 \pm 5 \mathrm{~mm} \mathrm{Hg}$ and TPR to $419 \pm 153$ dynes $\cdot \mathrm{s} \cdot \mathrm{cm}^{-5}$, respectively (both $P<.0005$ ). The cardiac index had increased to $2.52 \pm 0.56 \mathrm{~L} \cdot \mathrm{min} \cdot \mathrm{m}^{-2}(P<.002)$. Postoperatively, residual pulmonary arterial hypertension
(mPAP > $25 \mathrm{~mm} \mathrm{Hg}$; range 26-35 $\mathrm{mm} \mathrm{Hg}$ ) was observed in 10 patients, in 5 of whom mPAP was above $30 \mathrm{~mm} \mathrm{Hg}$.

The postoperative course was uncomplicated in all but 3 patients. One patient had severe pneumonia, because of which she required mechanical ventilation for 9 days. Two other patients had mild reperfusion lung injury and required mechanical ventilation for 3 and 5 days, respectively. Median duration of mechanical ventilation was 1 day (range 1-9 days); median intensive care unit stay was 4 days (range 1-13 days).

In the patients with CTEPH, before PEA, RVEDV and RVESV were increased as compared with the healthy controls (Figure 1). In contrast, LVEDV was decreased, whereas LVESV did not differ (Figure 1). After PEA, RV dimensions normalized. Both RVEDV and RVESV decreased significantly (Figures 1 and 2). In fact, after PEA, RVEDV and RVESV did not differ from the healthy controls. In addition, after PEA, RVEF and RVSV increased significantly but did not completely normalize (Table 1). Postoperative MRI characteristics of the LV showed improvement of the LV function. LVEDV increased, whereas LVESV remained unchanged (Figures 1 and 2). As a consequence, LVEF and LVSV both normalized (Table 1).

Preoperatively, RV volumes, that is, RVEDV and RVESV, did not correlate with the hemodynamic severity of disease. However, RVEF correlated significantly with the TPR (Figure 3, A). Moreover, the observed change after PEA in the RVEF correlated with the change in TPR ( $r=$ $0.50, P<.05$; Spearman rank correlation test). 


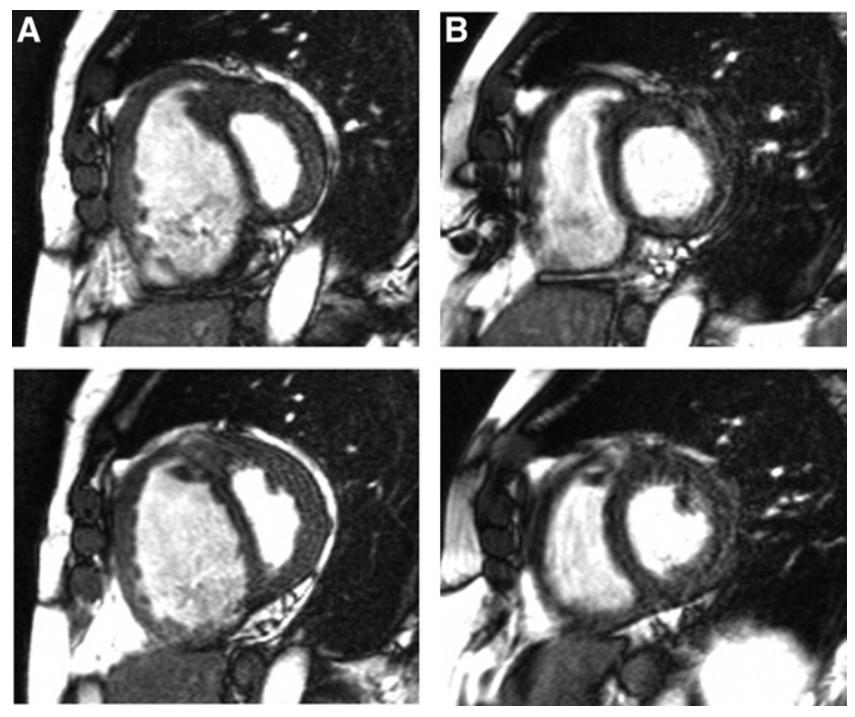

Figure 2. MRI short-axis cine images at basal (upper panel) and midventricular (lower panel) level, before (A) and after (B) PEA (relative time $55 \%$ in the cardiac cycle). Note the encroachment of the interventricular septum into the LV before PEA and the restoration of the septal bowing to normal after PEA. Note also the reversal of $\mathrm{RV}$ hypertrophy and the normalization of the $\mathrm{RV}$ and LV volumes.

The preoperatively demonstrated hypertrophy of the RV decreased after PEA but did not fully normalize in all patients (Figures 2 and $4, A$ and $B$ ). The preoperative and postoperative LV mass did not differ between patients with CTEPH and healthy controls (Figure 4, C).

Preoperatively, RV mass correlated with the TPR (Figure $3, B)$. Moreover, the change in RV mass after PEA correlated with the change in TPR $(r=0.63, P<.008$; Spearman

TABLE 1. RV and LV function in patients with CTEPH $(\mathbf{n}=$ 17) before and after PEA compared with healthy controls (n $=12$ )

\begin{tabular}{lccc}
\hline & \multicolumn{2}{c}{ CTEPH } & \\
\cline { 2 - 3 } & Before PEA & After PEA & Healthy controls \\
\hline RVSV, $\mathrm{mL} \cdot \mathrm{m}^{-2}$ & $26 \pm 7$ & $36 \pm 8 \S$ & $45 \pm 10 \| /{ }^{*}$ \\
RVEF, \% & $34 \pm 12$ & $56 \pm 7 \S$ & $67 \pm 9 \| / \dagger$ \\
$\mathrm{LVSV}, \mathrm{mL} \cdot \mathrm{m}^{-2}$ & $26 \pm 7$ & $37 \pm 8 \S$ & $45 \pm 9 \pm / \mathrm{ns}$ \\
$\mathrm{LVEF}, \%$ & $55 \pm 11$ & $66 \pm 5 \dagger$ & $69 \pm 8 \S / \mathrm{ns}$ \\
\hline
\end{tabular}

Data are shown as mean \pm standard deviation. $R V$, Right ventricular; $L V$, left ventricular; CTEPH, chronic thromboembolic pulmonary hypertension; $\angle V S V$, left ventricular stroke volume; $L V E F$, left ventricular ejection fraction. Differences in patients with CTEPH before and after PEA analyzed by Wilcoxon signed rank test; differences between patients with CTEPH and healthy controls (before/after PEA) analyzed by Mann-Whitney $U$ test. $* P<$ $.05 ; \dagger P<.005 ; \ddagger P<.001, \S P<.0005, \| P<.0001 ; n s$, no significant difference.
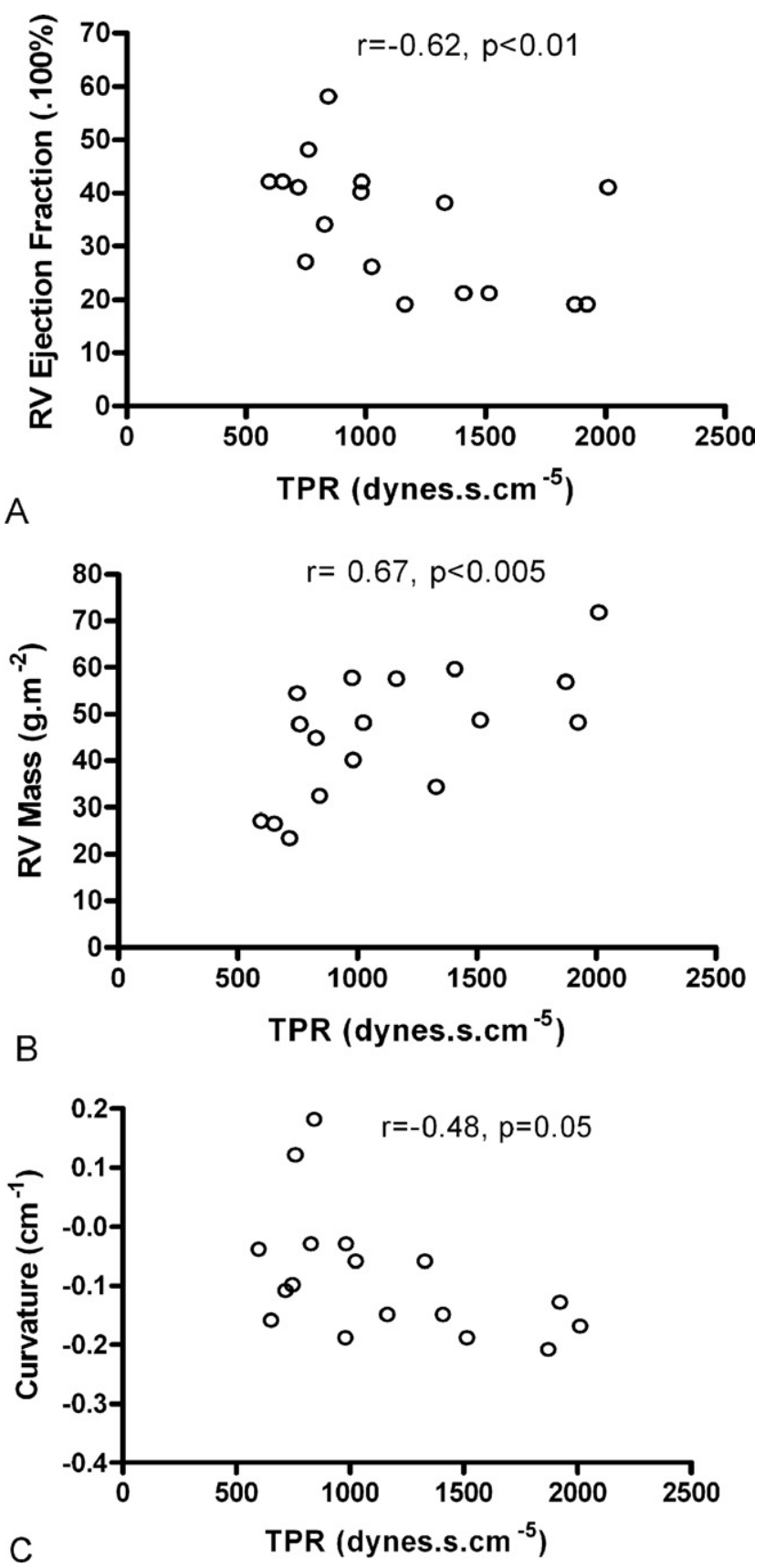

Figure 3. Correlation between RVEF (A), RV mass (B), and septal bowing (curvature: 1/R) (C) and the TPR in patients with CTEPH (n = 17) before PEA.

rank correlation test), as well as the change in mPAP $(r=$ $0.53, P<.05)$. In addition, RV mass tended to be higher in patients with residual pulmonary hypertension than in patients with normalized hemodynamics $(34 \pm 9$ vs $26 \pm 5 \mathrm{~g}$ $\left.\cdot \mathrm{m}^{-2}, P=.05\right)$.

LVSB was present in 15 patients with CTEPH before PEA. The mean septal bowing was $-0.09 \pm 0.11 \mathrm{~cm}^{-1}$. 


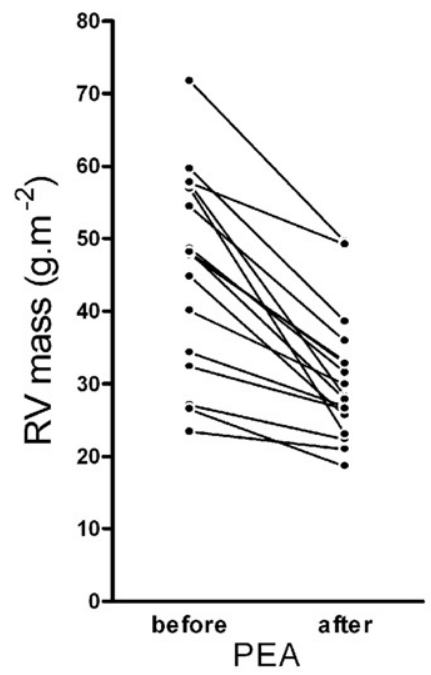

A
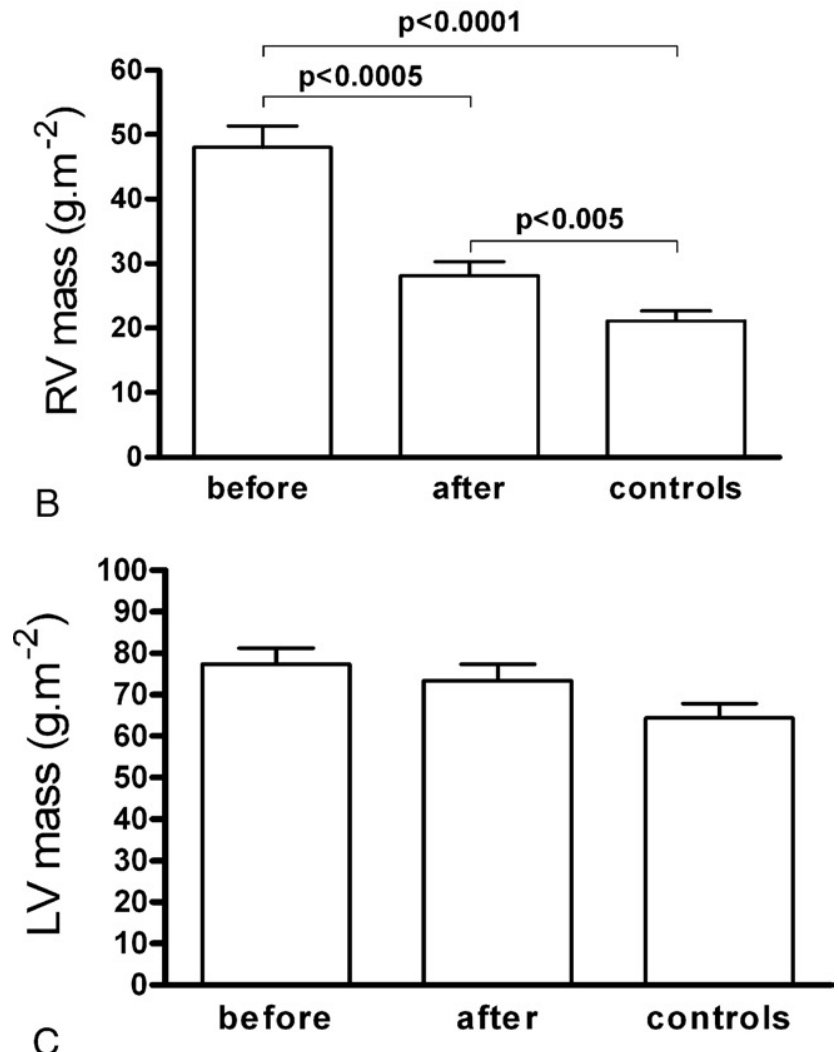

Figure 4. $R V(A, B)$ and $L V$ mass $(C)$ in patients with CTEPH $(n=$ 17) before and after PEA compared with healthy controls $(n=12)$. Data are expressed as mean \pm SEM.

The septal bowing normalized after PEA (Figures 2 and 5). Preoperatively, the (leftward ventricular) septal bowing correlated significantly with the TPR (Figure 3,C) and also the change in septal bowing after PEA correlated significantly with the change in TPR $(r=0.50, P<.05$; Spearman rank correlation test).

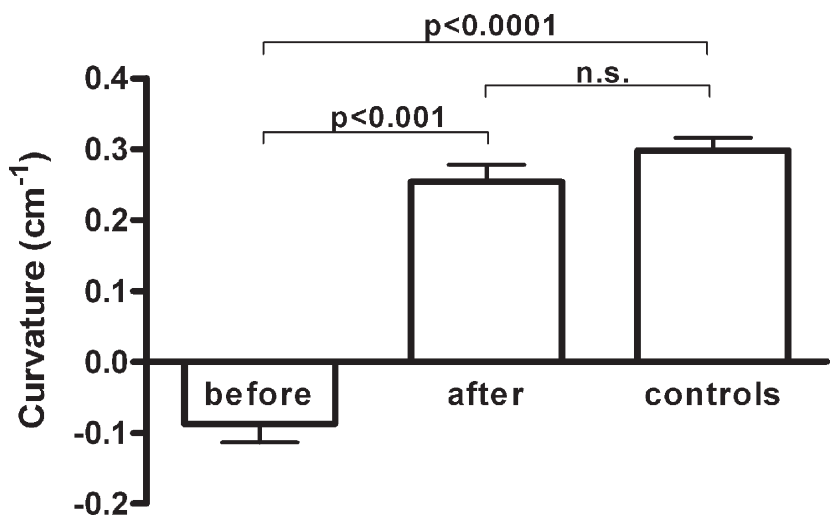

Figure 5. Septal bowing (curvature: 1/R) in patients with CTEPH ( $n=17$ ) before and after PEA compared with healthy controls ( $n$ $=12$ ). Data are expressed as mean \pm SEM.

\section{Discussion}

In the present study we demonstrated in patients with $\mathrm{CTEPH}$ that the observed RV remodeling (RV dilatation, hypertrophy, and LVSB) reversed after PEA. Moreover, the decrease of the level of RV hypertrophy and LVSB correlated significantly with the decrease in TPR after PEA.

Advanced pulmonary hypertension, as can be observed in CTEPH, is associated with chronic volume overload of the RV. ${ }^{14} \mathrm{RV}$ overload induces hypertrophy of the RV cardiac myocytes. MRI may be used to identify signs of chronic volume overload and RV hypertrophy. Our findings of the increased RV mass are in line with a study of Katz and coworkers, ${ }^{9}$ who quantified RV and LV mass in 12 patients with idiopathic pulmonary hypertension and 10 healthy controls. In the present study, after PEA the RV mass in patients with CTEPH decreased significantly but did not normalize completely. This might be explained at least in part by the fact that residual pulmonary hypertension was present in some patients. In these patients also the highest postoperative RV mass was observed. Although limited by the small number of patients included, this observation may suggest that RV mass as determined by MRI may be of use to assess postoperative outcome in patients with CTEPH.

In the present study, LVSB was present in the majority of patients. Early diastolic septal bowing is an ominous sign in patients with pulmonary hypertension. During systole, pressure in the LV usually exceeds the RV pressure, showing a (positive) curvature away from the LV center. During early $\mathrm{LV}$ diastole, the LV pressure drops to near zero to enable rapid LV filling. The RV pressure pushes the septum away from the RV center, causing (negative) LVSB.${ }^{15}$ Recently, $\mathrm{we}^{10}$ have demonstrated that the interventricular septal bowing correlated with severity of pulmonary hypertension in idiopathic pulmonary hypertension. In the present study, 
we demonstrated that also in CTEPH severity of disease (TPR) correlated with the septal bowing. Moreover, we also demonstrated that the change in septal bowing after PEA correlated with the observed hemodynamic improvement.

In contrast to Kreitner and coworkers, ${ }^{6}$ who described restoration of RV function 14 days after PEA, we studied cardiac morphology and function at least 4 months after PEA. In line with Kreitner's group, ${ }^{6}$ postoperatively, we observed an increase of the RVEF and stroke volume. In contrast, preoperatively, compared with the healthy controls, we demonstrated a decreased LV stroke volume and EF, which both normalized after the PEA procedure. The impairment of the LV function might be attributable to ventricular interaction or ventricular interdependence (also known as the "reverse Bernheim phenomenon"): RV dilation and hypertrophy shift the interventricular septum leftward, thereby causing decreased LV cavity size, contractility, compliance, and EF. ${ }^{16} \mathrm{LV}$ diastolic dysfunction, however, may also be caused in part by myocardial hypertrophy of the RV and interventricular septum, as documented earlier in patients with idiopathic pulmonary hypertension. ${ }^{15}$ Recently, we ${ }^{17}$ have demonstrated that ventricular interaction mediated by interventricular septal bowing caused impairment of the LV filling and thereby contributed to decreased stroke volumes observed in patients with pulmonary arterial hypertension. Our observations thus confirm and extend the notion that the impaired LV function in pulmonary arterial hypertension appears to be the consequence of the RV dysfunction and that, after hemodynamically successful PEA, LV function normalizes owing to the restoration of pulmonary hemodynamics and $\mathrm{RV}$ function.

The most widely noninvasive tool used in clinical practice to study RV dysfunction is echocardiography. In CTEPH, echocardiographically, restoration of RV function after PEA was reported before. ${ }^{18-20}$ In fact, improvement of RV geometry and LV diastolic function after PEA was already reported by Dittrich and coworkers in 1988 and $1989 .{ }^{21,22}$ In addition, reverse RV remodeling, that is, improvement of $\mathrm{RV}$ dimensions and $\mathrm{EF}$, was demonstrated in patients with idiopathic pulmonary hypertension after lung transplantation. ${ }^{23,24}$ The usefulness of echocardiography in this respect, however, is limited because of its technical limitations (acoustic window) and the absence of a reliable mathematical assumption due to complex geometry of the $\mathrm{RV}$. In view of these limitations, MRI is considered a superior imaging technique to quantify the characteristics of $\mathrm{RV}$ function and morphology. ${ }^{25-27}$ To our knowledge, this is the first study to demonstrate reverse RV remodeling in patients with CTEPH after PEA by means of MRI.

There are some limitations of the study. First, postoperative invasive measurements were determined immediately postoperatively, whereas MRI studies were performed at least 4 months after PEA. In view of earlier studies ${ }^{28,29}$ that showed that pulmonary artery pressure and TPR may further decrease during follow-up, it would have been more accurate to determine the hemodynamic parameters at the time of MRI. However, since the aim of the present study was to demonstrate restoration of $\mathrm{RV}$ remodeling in patients after successful PEA, for ethical reasons, we choose not to do so. Nevertheless, the level of restoration of RV remodeling correlated significantly with parameters of direct postoperative hemodynamic outcome. In view of these limitations, however, our observations need to be confirmed in future studies.

Second, although MRI is an excellent tool to study cardiac remodeling, it also has its limitations to assess the complex RV anatomy. In measuring the RV volume, the contouring of the most basal RV slice has a large effect. While the image plane is fixed, the RV base moves through-plane during the cardiac cycle. This can make it difficult for the observer to assign a region to the RV, the right atrium, or the pulmonary artery. The RV wall can be distinguished from the right atrial wall, however, by its thicker and trabeculated surface. Also, the cine mode of the basal slice can be used: if the tricuspid valve moves through the image plane during systole, this indicates that in end-diastole the upper posterior part of the RV is in the image plane, whereas in end-systole the right atrium has moved into the plane. Similarly, observation of the pulmonary valve during cine is used to separate the upper anterior part of the RV from the pulmonary artery lumen. In addition, by measuring RV mass, the contouring of the trabeculated endocardial border is observer dependent. While the endocardial contour in both the end-diastolic and end-systolic frames is being drawn, the small trabeculae are systematically excluded from the myocardium. $\mathrm{RV}$ mass is then calculated by the average of RV enddiastolic and end-systolic mass. By meticulous application of the above, assessment of RV mass, volumes, and function by breath-hold cine MRI, however, is accurate and highly reproducible. ${ }^{30}$

In conclusion, in this study we demonstrated that RV remodeling observed in patients with CTEPH was restored after a hemodynamically successful PEA. Moreover, the level of restoration of remodeling after PEA was demonstrated to correlate with the observed hemodynamic improvement. MRI appears to be a valuable tool to evaluate cardiac remodeling and function in patients with CTEPH both before and during follow-up after pulmonary thromboendarterectomy.

\section{References}

1. Fedullo PF, Auger WR, Kerr KM, Rubin LJ. Chronic thromboembolic pulmonary hypertension. $N$ Engl J Med. 2001;345:1465-72.

2. Jamieson SW, Kapelanski DP. Pulmonary endarterectomy. Curr Probl Surg. 2000;37:165-252. 
3. Riedel M, Stanek V, Widimsky J, Prerovsky I. Longterm follow-up of patients with pulmonary thromboembolism. Late prognosis and evolution of hemodynamic and respiratory data. Chest. 1982;81:151-8.

4. Jamieson SW, Kapelanski DP, Sakakibara N, Manecke GR, Thistlethwaite PA, Kerr KM, et al. Pulmonary endarterectomy: experience and lessons learned in 1,500 cases. Ann Thorac Surg. 2003;76:1457-62.

5. Mo M, Kapelanski DP, Mitruka SN, Auger WR, Fedullo PF, Channick $\mathrm{RN}$, et al. Reoperative pulmonary thromboendarterectomy. Ann Thorac Surg. 1999;68:1770-6.

6. Kreitner KFJ, Ley S, Kauczor HU, Mayer E, Kramm T, Pitton MB, et al. Chronic thromboembolic pulmonary hypertension: pre- and postoperative assessment with breath-hold MR imaging techniques. Radiology. 2004;232:535-43.

7. Ley S, Kramm T, Kauczor HU, Mayer E, Heussel CP, Thelen M, et al. Pre- and postoperative assessment of hemodynamics in patients with chronic thromboembolic pulmonary hypertension by MR techniques. Rofo. 2003;175:1647-54

8. Hoeper MM, Tongers J, Leppert A, Baus S, Maier R, Lotz J. Evaluation of right ventricular performance with a right ventricular ejection fraction thermodilution catheter and MRI in patients with pulmonary hypertension. Chest. 2001;120:502-7.

9. Katz J, Whang J, Boxt LM, Barst RJ. Estimation of right ventricular mass in normal subjects and in patients with primary pulmonary hypertension by nuclear magnetic resonance imaging. J Am Coll Cardiol. 1993;21:1475-81.

10. Roeleveld RJ, Marcus JT, Faes TJC, Gan TJ, Boonstra A, Postmus PE, et al. Interventricular septal configuration at MR imaging and pulmonary arterial pressure in pulmonary hypertension. Radiology. 2005; 234:710-7.

11. Auger WR, Fedullo PF, Moser KM, Buchbinder M, Peterson KL. Chronic major-vessel thromboembolic pulmonary artery obstruction: appearance at angiography. Radiology. 1992;182:393-8.

12. Alfakih K, Plein S, Thiele H, Jones T, Ridgway JP, Sivananthan MU. Normal human left and right ventricular dimensions for MRI as assessed by turbo gradient echo and steady-state free precession imaging sequences. J Magn Reson Imaging. 2003;17:323-9.

13. Fedullo PF, Auger WR, Channick RN, Kerr KM, Rubin LJ. Chronic thromboembolic pulmonary hypertension. Clin Chest Med. 2001;22: 561-81.

14. Nagaya N, Nishikimi T, Okano Y, Uematsu M, Satoh T, Kyotani S, et al. Plasma brain natriuretic peptide levels increase in proportion to the extent of right ventricular dysfunction in pulmonary hypertension. J Am Coll Cardiol. 1998;31:202-8.

15. Marcus JT, Vonk Noordegraaf A, Roeleveld RJ, Postmus PE, Heethaar RM, Van Rossum AC, et al. Impaired left ventricular filling due to right ventricular pressure overload in primary pulmonary hypertension: noninvasive monitoring using MRI. Chest. 2001;119:1761-5

16. Alpert JS. The effect of right ventricular dysfunction on left ventricular form and function. Chest. 2001;119:1632-3.

17. Gan TJ, Lankhaar JW, Marcus JT, Westerhof N, Marques KM, Bronzwaer JG, et al. Impaired left ventricular filling due to right to left ventricular interaction in patients with pulmonary arterial hypertension. Am J Physiol Heart Circ Physiol. 2006;290:H1528-33.

18. Menzel T, Wagner S, Kramm T, Mohr-Kahaly S, Mayer E, Braeuninger $\mathrm{S}$, et al. Pathophysiology of impaired right and left ventricular function in chronic embolic pulmonary hypertension: changes after pulmonary thromboendarterectomy. Chest. 2000;118: 897-903.

19. Menzel T, Kramm T, Bruckner A, Mohr-Kahaly S, Mayer E, Meyer J. Quantitative assessment of right ventricular volumes in severe chronic thromboembolic pulmonary hypertension using transthoracic threedimensional echocardiography: changes due to pulmonary thromboendarterectomy. Eur J Echocardiogr. 2002;3:67-72.

20. Menzel T, Kramm T, Mohr-Kahaly S, Mayer E, Oelert H, Meyer J. Assessment of cardiac performance using Tei indices in patients undergoing pulmonary thromboendarterectomy. Ann Thorac Surg. 2002; 73:762-6.

21. Dittrich HC, Nicod PH, Chow LC, Chappuis FP, Moser KM, Peterson KL. Early changes of right heart geometry after pulmonary thromboendarterectomy. J Am Coll Cardiol. 1988;11:937-43.

22. Dittrich HC, Chow LC, Nicod PH. Early improvement in left ventricular diastolic function after relief of chronic right ventricular pressure overload. Circulation. 1989;80:823-30.

23. Kasimir MT, Seebacher G, Jaksch P, Winkler G, Schmid K, Marta GM, et al. Reverse cardiac remodelling in patients with primary pulmonary hypertension after isolated lung transplantation. Eur J Cardiothorac Surg. 2004;26:776-81.

24. Vizza CD, Lynch JP, Ochoa LL, Richardson G, Trulock EP. Right and left ventricular dysfunction in patients with severe pulmonary disease. Chest. 1998;113:576-83

25. Helbing WA, Rebergen SA, Maliepaard C, Hansen B, Ottenkamp J, Reiber JHC, et al. Quantification of right ventricular function with magnetic resonance imaging in children with normal hearts and with congenital heart disease. Am Heart J. 1995;130:828-37.

26. Helbing WA, Bosch HG, Maliepaard C, Rebergen SA, van der Gees RJ, Hansen B, et al. Comparison of echocardiographic methods with magnetic resonance imaging for assessment of right ventricular function in children. Am J Cardiol. 1995;76:589-94.

27. Vogel M, Gutberlet M, Dittrich S, Hosten N, Lange PE. Comparison of transthoracic three dimensional echocardiography with magnetic resonance imaging in the assessment of right ventricular volume and mass. Heart. 1997;78:127-30.

28. Mayer E, Dahm M, Hake U, Schmid FX, Pitton M, Kupferwasser I, et al. Mid-term results of pulmonary thromboendarterectomy for chronic thromboembolic pulmonary hypertension. Ann Thorac Surg. 1996;61: 1788-92.

29. Zoia MC, D'Armini AM, Beccaria M, Corsico A, Fulgoni P, Klersy $\mathrm{C}$, et al. Mid term effects of pulmonary thromboendarterectomy on clinical and cardiopulmonary function status. Thorax. 2002;57:60812 .

30. Beygui F, Furber A, Delepine S, Prunier F, Helft G, Metzger JP, et al. Assessment of biventricular remodeling by magnetic resonance imaging after successful primary stenting for acute myocardial infarction. Am J Cardiol. 2004:94:354-7. 